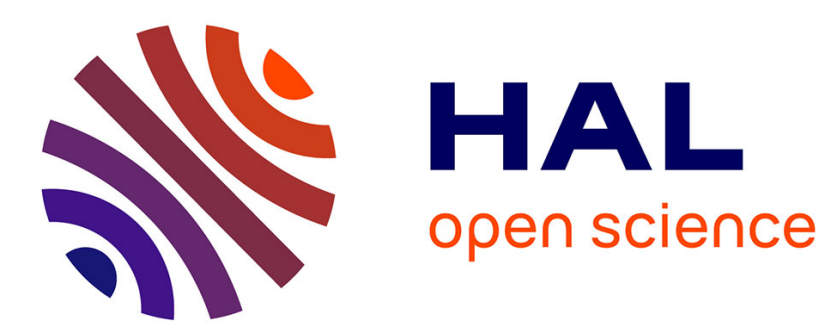

\title{
An overlapping theorem with applications
}

Javier Cilleruelo, Gérald Tenenbaum

\section{- To cite this version:}

Javier Cilleruelo, Gérald Tenenbaum. An overlapping theorem with applications. Primeras Jornadas de Teoría de Números, 2007, Spain. pp.107-118. hal-00091304v2

\section{HAL Id: hal-00091304 \\ https://hal.science/hal-00091304v2}

Submitted on 19 Feb 2007

HAL is a multi-disciplinary open access archive for the deposit and dissemination of scientific research documents, whether they are published or not. The documents may come from teaching and research institutions in France or abroad, or from public or private research centers.
L'archive ouverte pluridisciplinaire HAL, est destinée au dépôt et à la diffusion de documents scientifiques de niveau recherche, publiés ou non, émanant des établissements d'enseignement et de recherche français ou étrangers, des laboratoires publics ou privés. 


\title{
AN OVERLAPPING THEOREM WITH APPLICATIONS
}

\author{
JAVIER CILLERUELO AND GÉRALD TENENBAUM
}

\begin{abstract}
We establish a general and optimal lower bound for the complete sum of the probabilities of $k$-intersections of $n$ events. We then describe various applications to additive and multiplicative number theory, graph theory, coding theory, study of lattice points on circles, and divisors of polynomials
\end{abstract}

\section{INTRODUCTION}

Let $\mu$ be a positive measure on a set $\Omega,\left\{E_{j}\right\}_{j=1}^{k}$ a family of measurable subsets, and set

$$
\tau_{m}:=\sum_{1 \leqslant j_{1}<\cdots<j_{m} \leqslant k} \mu\left(E_{j_{1}} \cap \cdots \cap E_{j_{m}}\right) \quad(m \geqslant 1) .
$$

We address here the problem of obtaining lower bounds for $\tau_{m}$ in terms of $\tau_{1}$. For $m \geqslant 2$, the quantity $\tau_{m}$ may be thought of as the global amount of $m$-overlapping in the family $\left\{E_{j}\right\}_{j=1}^{k}$.

Many problems in Combinatorial Number Theory may be tackled by using estimates for $\tau_{m}$. According to the specific situation under consideration, appropriate choices of the set $\Omega$, the family of subsets $\left\{E_{j}\right\}_{j=1}^{k}$ and the measure $\mu$ may be performed.

The integer parameter $m \geqslant 1$ being fixed, our results will be conveniently described in terms of the continuous, piecewise linear, interpolation of the binomial coefficients $\left(\begin{array}{c}n \\ m\end{array}\right)(n \in \mathbb{N})$. Thus, we define

$$
Q_{m}(x):=\left(\begin{array}{c}
\lfloor x\rfloor \\
m
\end{array}\right)+\left(\begin{array}{c}
\lfloor x\rfloor \\
m-1
\end{array}\right)\langle x\rangle=\left(\begin{array}{c}
\lfloor x\rfloor \\
m
\end{array}\right)\langle x\rangle+\left(\begin{array}{c}
\lfloor x\rfloor+1 \\
m
\end{array}\right)(1-\langle x\rangle) \quad\left(x \in \mathbb{R}^{+}\right)
$$

where $\lfloor x\rfloor$ and $\langle x\rangle$ denote respectively the integer part and the fractional part of $x$. We have

$$
Q_{m}(x) \geqslant\left(\begin{array}{c}
x \\
m
\end{array}\right) \quad\left(x \in \mathbb{R}^{+}\right)
$$

for the right-hand side is a convex function of $x$, with equality when $x$ is an integer. We also note that $Q_{1}(x)=x$, and that, for all $m$, the function $Q_{m}$ is continuous, convex and satisfies $Q_{m}(x)=0$ whenever $x \leqslant m-1$.

We state our main result in a probabilistic setting.

Date: February 19, 2007.

1991 Mathematics Subject Classification. 60C05.

Key words and phrases. Probability of intersections, divisors, lattice points, Sidon sets, graph theory, divisors of polynomials, coding theory.

The authors take pleasure in thanking Bernardo López for helpful suggestions on the presentation of sections 1 and 2 . 
Theorem 1.1 (Overlapping Theorem). Let $(\Omega, \mathcal{A}, \mathbb{P})$ be a probability space and let $\left\{E_{j}\right\}_{j=1}^{k}$ denote a family of events. Write

$$
\sigma_{m}:=\sum_{1 \leqslant j_{1}<\cdots<j_{m} \leqslant k} \mathbb{P}\left(E_{j_{1}} \cap \cdots \cap E_{j_{m}}\right) \quad(m \geqslant 1) .
$$

Then we have

$$
\sigma_{m} \geqslant Q_{m}\left(\sigma_{1}\right)
$$

The case $m=2$ is essentially due to Gillis [6]. The general bound has been outlined by Klazar in [7]. By a different method, we prove, the above result in the next section, together with the fact that inequality (1.1) is optimal in its generality.

In section 3, we describe various applications. The results obtained there are not all new: our main purpose is to point out that they all allow a unified approach.

\section{The OVERLAPPING THEOREM}

We first prove Theorem 1.1. Put $f(\omega):=\sum_{1 \leqslant j \leqslant k} \mathbf{1}_{E_{j}}(\omega)$. Then

$$
(1+t)^{f(\omega)}=\prod_{1 \leqslant j \leqslant k}\left(1+\mathbf{1}_{E_{j}}(\omega) t\right) \quad(\omega \in \Omega) .
$$

Equating coefficients of $t^{m}$ on both sides, we obtain

$$
Q_{m}(f(\omega))=\left(\begin{array}{c}
f(\omega) \\
m
\end{array}\right)=\sum_{1 \leqslant j_{1}<\cdots<j_{m} \leqslant k} \mathbf{1}_{E_{j_{1}}} \mathbf{1}_{E_{j_{2}}} \cdots \mathbf{1}_{E_{j_{m}}} \quad(\omega \in \Omega) .
$$

Integrating with respect to $\mathrm{d} \mathbb{P}(\omega)$, we obtain $\sigma_{m}=\mathbb{E}\left(Q_{m}(f)\right)$.

Since $Q_{m}$ is convex, we may apply Jensen's inequality (see, e.g., [11], Theorem 3.3) to get

$$
Q_{m}\left(\sigma_{1}\right)=Q_{m}(\mathbb{E}(f)) \leqslant \mathbb{E}\left(Q_{m}(f)\right)=\sigma_{m}
$$

It is not difficult to see that Theorem 1.1 cannot be improved. Let $I=\mathbb{R} / \mathbb{Z}$ be equipped with the Haar measure. For given $0<\sigma<k \in \mathbb{N}$ and all integers $j$, $1 \leqslant j \leqslant k$, we define

$$
E_{j}:=\{x \in I: 0 \leqslant x+j / k<\sigma / k(\bmod 1)\},
$$

so that each $E_{j}$ has measure $\sigma / k$. Put $\nu=\lfloor\sigma\rfloor$. Then each $x \in I$ belongs to exactly $\nu$ or $\nu+1$ sets $E_{j}$, the latter case being excluded if $\sigma \in \mathbb{N}$. Thus

$$
f(x)=\sum_{1 \leqslant j \leqslant k} \mathbf{1}_{E_{j}}(x) \in\{\nu, \nu+1\} \quad(x \in I) .
$$

Writing $A_{\kappa}:=f^{-1}(\{\kappa\})$, we infer that

$$
\mathbb{E}(f)=\sigma=\nu \mathbb{P}\left(A_{\nu}\right)+(\nu+1) \mathbb{P}\left(A_{\nu+1}\right)=\nu+\mathbb{P}\left(A_{\nu+1}\right) .
$$

This implies in turn, with $w:=\mathbb{P}\left(A_{\nu+1}\right)=\sigma-\nu$,

$$
\mathbb{E}\left(Q_{m}(f)\right)=\left(\begin{array}{c}
\nu+1 \\
m
\end{array}\right) w+\left(\begin{array}{c}
\nu \\
m
\end{array}\right)(1-w)=Q_{m}(\sigma) .
$$

Actually, equality holds if and only if $f=\sum \mathbf{1}_{E_{j}}$ takes no more than two consecutive integer values and $\mathbb{E}(f)=\sigma$.

For most the applications, the next corollary, which is also optimal, is sufficient. 
Corollary 2.1. Let $(\Omega, \mathcal{A}, \mathbb{P})$ be a probability space and let $\left\{E_{j}\right\}_{j=1}^{k}$ denote a family of events. Then, for each $m \geqslant 1$, we have

$$
\left(\begin{array}{c}
k \\
m
\end{array}\right) \max _{1 \leqslant j_{1}<\cdots<j_{m} \leqslant k} \mathbb{P}\left(E_{j_{1}} \cap \cdots \cap E_{j_{m}}\right) \geqslant Q_{m}\left(\sigma_{1}\right) .
$$

Proof. This is obvious since $\sigma_{m}$ is a sum with $\left(\begin{array}{c}k \\ m\end{array}\right)$ summands.

We now proceed with our optimality assertion.

Theorem 2.2. Let $0<\sigma \leqslant k \in \mathbb{N}$. There exist a probability space and a sequence of events $\left\{E_{j}\right\}_{j=1}^{k}$ such that every intersection

$$
E_{j_{1}} \cap \cdots \cap E_{j_{m}}, \quad 1 \leqslant j_{1}<\cdots<j_{m} \leqslant k
$$

has probability $Q_{m}(\sigma) /\left(\begin{array}{c}k \\ m\end{array}\right)$.

Proof. Let $k \in \mathbb{N}, A:=[1, k] \cap \mathbb{N}, \Omega:=\mathcal{P}(A)$, and define

$$
E_{j}:=\{J \subset A: j \in J\} \in \Omega \quad(1 \leqslant j \leqslant k) .
$$

Let $\nu:=\lfloor\sigma\rfloor, w:=\sigma-\nu$, and let $\Omega_{\nu}$ denote the subset of $\Omega$ comprising all sets $E$ with $\nu$ elements. Then, obviously,

$$
f(E)=\sum_{1 \leqslant j \leqslant k} \mathbf{1}_{E_{j}}(E)=\nu, \quad \sum_{j_{1}<\cdots<j_{m}} \mathbf{1}_{E_{j_{1}}}(E) \ldots \mathbf{1}_{E_{j_{m}}}(E)=\left(\begin{array}{c}
\nu \\
m
\end{array}\right) \quad\left(E \in \Omega_{\nu}\right) .
$$

Hence, selecting $\mathbb{P}$ as the uniform measure $\mu_{\nu}$ supported on $\Omega_{\nu}$,

$$
\sigma_{m}=\mathbb{E}\left(\left(\begin{array}{c}
f \\
m
\end{array}\right)\right)=\left(\begin{array}{c}
\nu \\
m
\end{array}\right) .
$$

Furthermore, by symmetry, all $E_{j_{1}} \cap \cdots \cap E_{j_{m}}$ have the same probability. By linear combination, the above is also true for $\mathbb{P}=w \mu_{\nu+1}+(1-w) \mu_{\nu}$. Therefore we get for this choice

$$
\sigma_{m}=w\left(\begin{array}{c}
\nu+1 \\
m
\end{array}\right)+(1-w)\left(\begin{array}{c}
\nu \\
m
\end{array}\right)=Q_{m}(\sigma)
$$

\section{Applications}

3.1. Primes. Our first application is an unusual proof of a well-known estimate for the sum of the reciprocals of primes.

Theorem 3.1. For $n \geqslant 3$, we have

$$
\sum_{p \leqslant n} \frac{1}{p} \leqslant \frac{\log \log (2 n+1)}{\log 2}+1
$$

Proof. Let $X$ denote the random variable defined by

$$
\mathbb{P}(X=r)= \begin{cases}1 / n & \text { for } 1 \leqslant r \leqslant n \\ 0 & \text { otherwise, }\end{cases}
$$

and, for each prime $p \leqslant n$, select $E_{p}:=\{\omega: X(\omega) \equiv 0(\bmod p)\}$. We have,

$$
\sigma_{m}=\sum_{p_{i_{1}}<\cdots<p_{i_{m}} \leqslant n} \mathbb{P}\left(E_{p_{i_{1}}} \cdots E_{p_{i_{m}}}\right)=\frac{1}{n} \sum_{p_{i_{1}}<\cdots<p_{i_{m}} \leqslant n}\left\lfloor\frac{n}{p_{i_{1}} \cdots p_{i_{m}}}\right\rfloor \leqslant \sum_{\substack{1 \leqslant r \leqslant n \\ \nu(r)=m}} \frac{1}{r} .
$$


where $\nu(r)$ denotes the number of prime factors of $r$. This also holds for $m=0$ if we set $\sigma_{0}=1$. Therefore

$$
2^{\sigma_{1}}=\sum_{m \geqslant 0}\left(\begin{array}{l}
\sigma_{1} \\
m
\end{array}\right) \leqslant \sum_{m \geqslant 0} \sigma_{m} \leqslant \sum_{1 \leqslant r \leqslant n} \frac{1}{r} \leqslant \log (2 n+1) .
$$

the stated bound follows, since we have from above

$$
\sigma_{1}=\frac{1}{n} \sum_{p \leqslant n}\left\lfloor\frac{n}{p}\right\rfloor \geqslant \sum_{p \leqslant n} \frac{1}{p}-1
$$

3.2. Graphs. The study of extremal problems in graph theory was initiated by Erdős and Turán. The theorem below was originally proved by Kővari, Sós and Turán [8]. The complete bipartite graph $K_{r, s}$ is a graph with two sets of vertices, one with $r$ members and one with $s$, such that each vertex in one set is adjacent to every vertex in the other set and to no vertex in its own set.

Theorem 3.2. Let $r, s$ be positive integers and $G$ be a graph with $n$ vertices containing no subgraph $K_{r, s}$. Then $G$ contains at most

$$
\frac{1}{2}(r-1)^{1 / s} n^{2-1 / s}+\frac{1}{2}(s-1) n
$$

edges.

Proof. Let $V$ be the set of vertices of $G$, so that $|V|=n$, and $E$ be the set of edges. Define a random variable $X: \Omega=V \rightarrow V$ with law $\mathbb{P}(X=v)=1 / n$ and, for each $v \in V$, let $E_{v}:=\{\omega:\{X(\omega), v\} \in E\}, \operatorname{deg}(v):=\left|E_{v}\right|$. Then $\mathbb{P}\left(E_{v}\right)=\operatorname{deg}(v) / n$ and

$$
\sigma_{1}=\frac{1}{n} \sum_{v \in E} \operatorname{deg}(v)=\frac{2|E|}{n}
$$

Since $G$ contains no subgraph of type $K_{r, s}$, we have $\mathbb{P}\left(E_{v_{1}} \cdots E_{v_{s}}\right) \leqslant(r-1) / n$ whenever the $E_{v_{j}}$ are pairwise distinct. Therefore,

$$
\sigma_{s} \leqslant \frac{r-1}{n}\left(\begin{array}{l}
n \\
s
\end{array}\right)
$$

Now we apply the overlapping theorem to obtain

$$
\frac{r-1}{n} \frac{n^{s}}{s !} \geqslant \frac{r-1}{n}\left(\begin{array}{c}
n \\
s
\end{array}\right) \geqslant \sigma_{s} \geqslant\left(\begin{array}{c}
\sigma_{1} \\
s
\end{array}\right)=\left(\begin{array}{c}
2|E| / n \\
s
\end{array}\right) \geqslant \frac{(2|E| / n-(s-1))^{s}}{s !}
$$

This yields the required inequality.

Remark. It is known that the constant $(r-1)^{1 / 2} / 2$ is sharp for $s=2$. 
3.3. Sidon sets. A set of integers $A$ is called a Sidon set if all sums

$$
a+a^{\prime} \quad\left(a \leqslant a^{\prime}, a \in A, a^{\prime} \in A\right)
$$

are distinct. A major problem in this theory consists in estimating the size $F(N)$ of the largest Sidon set contained in $\{1, \ldots, N\}$. Erdős [5] proved the upper bound

$$
F(N) \leqslant N^{1 / 2}+O\left(N^{1 / 4}\right)
$$

and Lindström [10] gave a more precise estimate (stated and proved below) which has not been improved in 37 years. Ruzsa [12] gave a new proof of it, using an easy but interesting lemma, which we prove via the overlapping theorem.

Lemma 3.3. Let $A$ and $B$ be two finite sets of integers. If $A$ is a Sidon set then

$$
|A+B| \geqslant \frac{|A|^{2}|B|}{|A|+|B|-1} .
$$

Proof. Let $X$ denote the integer random variable with law given by

$$
\mathbb{P}(X=m)= \begin{cases}1 /|A+B| & \text { if } m \in A+B \\ 0 & \text { otherwise. }\end{cases}
$$

For each $b \in B$ we set $E_{b}=\{X \in A+b\}$. Then $\mathbb{P}\left(E_{b}\right)=|A| /|A+B|$ and

$$
\sigma_{1}=\frac{|A||B|}{|A+B|}
$$

On the other hand, if $b \neq b^{\prime}, \mathbb{P}\left(E_{b} E_{b^{\prime}}\right) \leqslant 1 /|A+B|$, for $A$ is a Sidon set, whence $\sigma_{2} \leqslant\left(\begin{array}{c}|B| \\ 2\end{array}\right) /|A+B|$. Finally,

$$
\left(\begin{array}{c}
|B| \\
2
\end{array}\right) \frac{1}{|A+B|} \geqslant \sigma_{2} \geqslant Q_{2}\left(\sigma_{1}\right) \geqslant \frac{\sigma_{1}\left(\sigma_{1}-1\right)}{2}=\frac{|A||B|}{2|A+B|}\left(\frac{|A||B|}{|A+B|}-1\right),
$$

and the stated inequality follows.

Theorem 3.4 (Lindström). If $A \subset[1, N]$ is a Sidon set, then $|A| \leqslant N^{1 / 2}+N^{1 / 4}+1$.

Proof. Write $|A|=m$, take $B=\{1, \ldots, n\}$ with $n=\left\lfloor(m N)^{1 / 2}\right\rfloor+1$ and apply above lemma. We get

$$
N+(N m)^{1 / 2} \geqslant N+n-1 \geqslant|A+B| \geqslant \frac{m^{2} n}{m+n-1} \geqslant \frac{m^{2}(m N)^{1 / 2}}{m+(m N)^{1 / 2}},
$$

from which we derive that $m \leqslant\left(\sqrt{N^{1 / 2}+\frac{1}{4}}+\frac{1}{2}\right)^{2}<N^{1 / 2}+N^{1 / 4}+1$.

3.4. Coding theory. Write $Z_{q}:=[1, q] \cap \mathbb{N}$. The Hamming distance $d\left(w, w^{\prime}\right)$ of two words $w, w^{\prime} \in Z_{q}^{n}$ of length $n$, is the number of locations at which the letters from $w$ and $w^{\prime}$ are different. A classical problem in coding theory is to estimate the cardinality $A_{q}(n, d)$ of the largest code in $Z_{q}^{n}$ with given minimal Hamming distance $d$. Applying the overlapping theorem, we establish an upper bound for this problem, known as Plotkin bound.

Theorem 3.5 (Plotkin bound). Assume $q d>n(q-1)$. Then

$$
A_{q}(n, d) \leqslant \frac{q d}{q d-n(q-1)} \text {. }
$$


Proof. Let $\Omega:=\{(k, h): 1 \leqslant k \leqslant n, 0 \leqslant h \leqslant q-1\}$ and define a random variable $X$ such that

$$
\mathbb{P}(X=(k, h))=\frac{1}{n q} \text {. }
$$

Assume $C:=\left\{w_{i}: 1 \leqslant i \leqslant A_{q}(n, d)\right\}$ is a code such that $\min _{i \neq j} d\left(w_{i}, w_{j}\right)=d$. Writing $k_{i}$ for the $k$ th letter, or $k$-component, of $w_{i}$ we consider the events

$$
E_{i}:=\bigcup_{1 \leqslant k \leqslant n}\left\{X=\left(k, k_{i}\right)\right\} \quad\left(1 \leqslant i \leqslant A_{q}(d, n)\right) .
$$

Then $\mathbb{P}\left(E_{i}\right)=1 / q$ and $\sigma_{1}=A_{q}(n, d) / q$. Also

$$
E_{i} E_{j}=\bigcup_{1 \leqslant k \leqslant n}\left\{X=\left(k, k_{i}\right)=\left(k, k_{j}\right)\right\}
$$

So

Therefore,

$$
P\left(E_{i} E_{j}\right)=\frac{n-d\left(w_{i}, w_{j}\right)}{q n} \leqslant \frac{n-d}{q n} .
$$

$$
\left(\begin{array}{c}
A_{q}(n, d) \\
2
\end{array}\right) \frac{n-d}{q n} \geqslant \sigma_{2} \geqslant Q_{2}\left(\sigma_{1}\right) \geqslant\left(\begin{array}{c}
A_{q}(n, d) / q \\
2
\end{array}\right),
$$

and the required bound follows.

3.5. Divisors. Given integers $a_{1}, \ldots, a_{k}$, we denote by $\left(a_{1}, \ldots, a_{k}\right)$ their greatest common divisor.

Theorem 3.6. Let $\alpha \in] 0,1\left[, n \in \mathbb{N}^{*}\right.$ and $\left\{d_{j}\right\}_{j=1}^{k}$ a set of divisors of $n$ such that $\min _{1 \leqslant j \leqslant k} d_{j} \geqslant n^{\alpha}$. Then, for all $m \geqslant 1$, we have

$$
\max _{1 \leqslant j_{1}<\cdots<j_{m} \leqslant k}\left(d_{j_{1}}, \ldots, d_{j_{m}}\right)>n^{\alpha_{m}}
$$

where $\alpha_{m}:=Q_{m}(k \alpha) /\left(\begin{array}{c}k \\ m\end{array}\right)$.

Proof. Let $X$ denote the random variable defined by

$$
\mathbb{P}\left(X=p^{\nu}\right)=\frac{\log p}{\log n} \quad\left(p^{\nu} \mid n\right)
$$

Let $E_{j}=\left\{\omega: X \mid d_{j}\right\}$. Then $\mathbb{P}\left(E_{j}\right)=\left(\log d_{j}\right) / \log n$ and

$$
\mathbb{P}\left(E_{j_{1}} \cdots E_{j_{m}}\right)=\frac{\log \left(d_{j_{1}}, \ldots, d_{j_{m}}\right)}{\log n} .
$$

We observe that $\sigma_{1}=\sum_{j=1}^{k} \mathbb{P}\left(E_{j}\right) \geqslant k \alpha$. We may hence apply Corollary 2.1 to infer that there exist $d_{j_{1}}, \ldots, d_{j_{m}}$ such that

$$
\frac{\log \left(d_{j_{1}}, \ldots, d_{j_{m}}\right)}{\log n}=\mathbb{P}\left(E_{j_{1}} \cdots E_{j_{m}}\right) \geqslant Q_{m}\left(\sigma_{1}\right)\left(\begin{array}{c}
k \\
m
\end{array}\right)^{-1} \geqslant Q_{m}(k \alpha)\left(\begin{array}{c}
k \\
m
\end{array}\right)^{-1}
$$

For all values of $k, m$ and $\alpha$, the exponent $\alpha_{m}$ is optimal.

Theorem 3.7. For any positive integer $k$, and for any $\alpha, 0 \leqslant \alpha \leqslant 1$, there exists infinitely many integers $n$ with $k$ divisors $n^{\alpha}<d_{1}<\cdots<d_{k} \leqslant n$ and such that, for each $m, 2 \leqslant m \leqslant k$ and for any $d_{i_{1}}<\cdots<d_{i_{m}}$ we have $\left(d_{i_{1}}, \ldots, d_{i_{m}}\right) \leqslant n^{\alpha_{m}+o(1)}$, where $\alpha_{m}:=Q_{m}(k \alpha) /\left(\begin{array}{c}k \\ m\end{array}\right)$. 
Proof. The result is obtained in a straightforward manner by adapting the construction of Theorem 2.3. We omit the details.

The case $m=2$ was studied in [3], where the following result was stated.

Corollary 3.8. For $\alpha>0, k \in \mathbb{N}^{*}, \alpha_{2}=Q_{2}(k \alpha) /\left(\begin{array}{l}k \\ 2\end{array}\right)$, the interval $\left.] n^{\alpha}, n^{\alpha}+n^{\alpha_{2}}\right]$, contains at most, $k-1$ divisors of $n$.

Proof. Apply Theorem 3.6 for $m=2$, noticing that if $d_{i}, d_{j}$ belong to an interval $I$, then $\left(d_{i}, d_{j}\right) \leqslant|I|$.

Remark. It is an interesting and difficult problem to decide whether the exponent $\alpha_{2}$ in the corollary is sharp.

It is a natural problem to consider the divisors of an integer lying in an arithmetic progression. We give an easy proof of the following theorem of Lenstra [9]

Corollary 3.9 (Lenstra). Let $\alpha>1 / 4, n, q \in \mathbb{N}^{*}, q>n^{\alpha}$. Then, the number of divisors $d$ of $n$ such that $d \equiv a(\bmod q)$ is bounded by a function of $\alpha$ alone.

Proof. Write $q=n^{(1 / 4)+2 \varepsilon}$. We prove that the number of divisors in the form $d_{i}=a+m_{i} q$ lying in the interval $I_{r}=\left[n^{r \varepsilon}, n^{(r+1) \varepsilon}\right]$ is bounded by $1+1 / \varepsilon$ for each integer $r$ with $0 \leqslant r \leqslant 1 / \varepsilon$. This indeed implies that the total number of divisors in the arithmetic progression $a(\bmod q)$ is bounded by $(1+1 / \varepsilon)^{2}$.

Let $k$ be the number of divisors in $I_{r}$. Then, there exist $i, j$ such that

$$
n^{(r+1) \varepsilon} \geqslant d_{i}-d_{j}=q\left(m_{i}-m_{j}\right) \geqslant q\left(m_{i}, m_{j}\right) \geqslant q\left(d_{i}, d_{j}\right) \geqslant n^{(1 / 4)+2 \varepsilon} n^{Q_{2}(k r \varepsilon) /\left(\begin{array}{c}
k \\
2
\end{array}\right)} .
$$

Thus, $r \varepsilon+\varepsilon \geqslant \frac{1}{4}+2 \varepsilon+\{k r \varepsilon(k r \varepsilon-1)\} /\{k(k-1)\}$, which may be rewritten as

$$
\frac{1}{k-1} \geqslant \frac{(1 / 4)+\varepsilon}{r \varepsilon(1-r \varepsilon)}-1 \text {. }
$$

Since $r \varepsilon(1-r \varepsilon) \leqslant 1 / 4$, we obtain $k \leqslant 1+1 / \varepsilon$, as required.

The following result was suggested by R. de la Bretèche and was used in [1].

Corollary 3.10. Let $\varepsilon \in] 0,1]$ and $\alpha \in[0,1]$. For all $n \in \mathbb{N}^{*}$ and all a, $q$ such that $(a, q)=1, q>n^{\alpha-\alpha^{2}+2 \varepsilon}$, we have

$$
\mid\left\{d\left|n: d \equiv a(\bmod q), n^{\alpha}<d \leqslant n^{\alpha+\varepsilon}[\}\right| \leqslant\left(\alpha-\alpha^{2}+\varepsilon\right) / \varepsilon .\right.
$$

Proof. Let $d_{j}=a+m_{j} q(1 \leqslant j \leqslant k)$ be divisors of $n$ in $] n^{\alpha}, n^{\alpha+\varepsilon}$. From Theorem 3.6 with $m=2$, we see that

$$
\max _{1 \leqslant i<j \leqslant k}\left(d_{i}, d_{j}\right)>n^{\alpha_{2}} .
$$

However, we have $\left(d_{i}, d_{j}\right)=\left(m_{i} q+a,\left(m_{i}-m_{j}\right) q\right) \leqslant\left|m_{i}-m_{j}\right| \leqslant n^{\alpha+\varepsilon} / q \leqslant n^{\alpha^{2}-\varepsilon}$ for all $i, j$ with $i \neq j$. This is sufficient.

Changing the probability measure in Theorem 1.1, we get interesting variants of the above results. An example, given here without proof, is the following, where $\nu(d)$ denotes the number of distinct prime factors of $d$.

Theorem 3.11. Let Let $0<\alpha<1$ and $n \geqslant 1$. Assume $\left\{d_{j}\right\}_{j=1}^{k}$ is a set of distinct divisors of $n$ with $\nu\left(d_{j}\right)>\alpha \nu(n)$ for all $j$. Then

$$
\max _{1 \leqslant i<j \leqslant k} \nu\left(\left(d_{i}, d_{j}\right)\right)>\alpha_{2} \nu(n) .
$$


3.6. Lattice points on circles. It is known that the number of lattice points on the circle $x^{2}+y^{2}=n$ is not bounded uniformly in $n$. Schinzel proved that, on the circle $x^{2}+y^{2}=R^{2}$, an arc of length $R^{1 / 3}$ contains at most two lattice points. In [2], Córdoba and the first author proved a more general result for which we now provide a simpler proof using Theorem 1.1.

Theorem 3.12. Let $x^{2}+y^{2}=R^{2}$ be a circle, $k \in \mathbb{N}^{*}$, and $\gamma_{k}:=1 /(4[k / 2]+2)$. Then, an arc of length $R^{1 / 2-\gamma_{k}}$ contains at most $k$ lattice points.

Proof. Let $x^{2}+y^{2}=R^{2}=n=\prod_{1 \leqslant s \leqslant t}\left|\pi_{s}\right|^{2 m_{s}}$ be a circle, where the $\pi_{s} \in \mathbb{Z}[i]$ are Gaussian primes, and $m_{s} \in \mathbb{N}^{*}(1 \leqslant s \leqslant t)$. Assume that there are $k+1$ lattice points $\nu_{1}, \ldots, \nu_{k+1}$ of $\mathbb{Z}[i]$ on an arc of length $R^{\gamma}$. Let $X$ denote the random variable defined by

$$
\mathbb{P}\left(X=\bar{\pi}_{s}^{a}\right)=\mathbb{P}\left(X=\pi_{s}^{b}\right)=\log \left|\pi_{s}\right| / \log n \quad\left(1 \leqslant s \leqslant t, 1 \leqslant a, b \leqslant m_{s}\right) .
$$

For each $j \in[1, k+1]$ put $E_{j}:=\left\{X: X \mid \nu_{j}\right\}$. Then

$$
\mathbb{P}\left(E_{j}\right)=\frac{\log \left|\nu_{j}\right|}{\log n}=\frac{1}{2}
$$

and

$$
\mathbb{P}\left(E_{i} E_{j}\right)=\frac{\log \left|\left(\nu_{i}, \nu_{j}\right)\right|}{\log n} \leqslant \frac{\log \left|\nu_{i}-\nu_{j}\right|}{\log n}<\frac{\log R^{\gamma}}{\log n}=\frac{\gamma}{2} .
$$

Thus, $\sigma_{2}<\frac{1}{2}\left(\begin{array}{c}k+1 \\ 2\end{array}\right) \gamma$ and $\sigma_{1}=(k+1) / 2$. Therefore

$$
\left(\begin{array}{c}
k+1 \\
2
\end{array}\right) \frac{\gamma}{2}>\sigma_{2} \geqslant Q_{2}\left(\sigma_{1}\right) \geqslant Q_{2}\left(\frac{k+1}{2}\right),
$$

and so $\gamma>2 Q_{2}\left(\frac{k+1}{2}\right) /\left(\begin{array}{c}k+1 \\ 2\end{array}\right)=\frac{1}{2}-1 /(4[k / 2]+2)$.

We do not know whether the number of lattice points on arcs of length $R^{1 / 2}$ can be bounded independently of $R$. The above theorem yields that the number of lattice points on such $\operatorname{arcs}$ is $\ll \log R$.

3.7. Polynomials. The overlapping theorem may be used to provide an alternative proof of the following result, due to Jiménez and the first author [4].

Theorem 3.13. Let $\gamma>0, M(x) \in \mathbb{Z}[x]$ and $F_{1}(x), \ldots, F_{k}(x)$ be $k$ divisors of $M(x)$ in $\mathbb{Z}[x]$ such that $\min _{1 \leqslant j \leqslant k} \operatorname{deg} F_{j} \geqslant \gamma \operatorname{deg} M$. Then there exist $i, j \in[1, k]$, $i \neq j$, such that

$$
\operatorname{deg}\left(F_{i}-F_{j}\right) \geqslant(\operatorname{deg} M) Q_{2}(k \gamma)\left(\begin{array}{l}
k \\
2
\end{array}\right)^{-1} \geqslant \operatorname{deg} M\left\{\gamma^{2}-\frac{\gamma(1-\gamma)}{k-1}\right\}
$$

Proof. Write $M=p_{1}^{\alpha_{1}} \cdots p_{t}^{\alpha_{t}}$ a decomposition of $M(x)$ as a product of irreducible factors in $\mathbb{Z}[x]$. Let $X$ denote the random variable defined by

$$
\mathbb{P}\left(X=p_{s}^{\alpha}\right)=\frac{\operatorname{deg} p_{s}}{\operatorname{deg} M} \quad\left(1 \leqslant s \leqslant t, 1 \leqslant \alpha \leqslant \alpha_{s}\right) .
$$

Let $E_{j}=\left\{\omega: X \mid F_{j}(x)\right\}$. Then $\mathbb{P}\left(E_{j}\right)=\left(\operatorname{deg} F_{j}\right) / \operatorname{deg} M \geqslant \gamma$. By Corollary 2.1, there exist distinct indices $i, j$ such that

$$
\mathbb{P}\left(E_{i} E_{j}\right) \geqslant Q_{2}\left(\sigma_{1}\right)\left(\begin{array}{l}
k \\
2
\end{array}\right)^{-1} \geqslant Q_{2}(k \gamma)\left(\begin{array}{l}
k \\
2
\end{array}\right)^{-1} \geqslant \gamma^{2}-\frac{\gamma(1-\gamma)}{k-1}
$$


We complete the proof by observing that

$$
\mathbb{P}\left(E_{i} E_{j}\right)=\frac{\operatorname{deg}\left(F_{j}, F_{i}\right)}{\operatorname{deg} M} \leqslant \frac{\operatorname{deg}\left(F_{i}-F_{j}\right)}{\operatorname{deg} M} .
$$

\section{REFERENCES}

[1] R. de la Bretèche, Nombre de valeurs polynomiales qui divisent un entier, Math. Proc. Cambridge Philos. Soc. 131 (2001), no. 2, 193-209.

[2] J. Cilleruelo, A. Córdoba, Trigonometric polynomials and lattice points, Proc. Amer. Math. Soc. 115 (1992), no. 4, 899-905.

[3] J. Cilleruelo, J. Jiménez-Urroz, The hyperbola $x y=N$, J. Théor. Nombres Bordeaux 12 (2000), no. $1,87-92$

[4] J. Cilleruelo, J. Jiménez-Urroz, Divisors in a Dedekind domain, Acta Arith 85 (1998), no. 3, 229-233.

[5] P. Erdős, P. Turán, On a problem of Sidon in additive number theory, and on some related problems, J. London Math. Soc. 16 (1941) 212-215.

[6] J. Gillis, Note on a property of measurable sets, Journal London Math. Soc. 11 (1936), 139141.

[7] M. Klazar, Comments on a result of Trotter and Winkler in combinatorial probability, Paul Erds and his mathematics (Budapest, 1999), 127-129, Jnos Bolyai Math. Soc., Budapest, 1999.

[8] T. Kővari, V. T. Sós, P. Turán, On a problem of K. Zarankiewicz, Colloquium Math 3 (1954) $50-57$.

[9] H.W. Lenstra, Divisors in residue classes, Mathematics of computation 42 (1984), no. 165, $331-340$.

[10] B. Lindström, On a combinatorial problem in number theory, Canad. Math. Bull. 8 (1965) 477-490.

[11] W. Rudin, Real and complex analysis, Third edition, McGraw-Hill, 1987.

[12] I. Z. Ruzsa, Solving a linear equation in a set of integers, I, Acta Arith, 65 (1993), no. 3, $259-282$.

Javier Cilleruelo, Departamento de Matemáticas, Unversidad Autónoma de Madrid, MADRID 28049, ESPAÑA.

E-mail address: franciscojavier.cilleruelo@uam.es

Gérald Tenenbaum, Institut Élie Cartan, Université Henri Poincaré, B.P. 239, 54506 Vandeuvre-LÈs-Nancy, France.

E-mail address: gerald.tenenbaum@iecn.u-nancy.fr 